\title{
A Survey Approach on ECG Feature Extraction Techniques
}

\author{
Shalini Sahay \\ Research Sholar \\ RGPV, Bhopal
}

\author{
A.K.Wadhwani, PhD \\ Professor \& Head of EE \\ M.I.T.S, Gwalior
}

\author{
Sulochana Wadhwani, PhD \\ Professor in EE Deptt. \\ M.I.T.S, Gwalior
}

\begin{abstract}
ECG Feature Extraction plays a significant role in diagnosing most of the cardiac diseases. One cardiac cycle in an ECG signal consists of the P-QRS-T waves. This feature extraction scheme determines the amplitudes and intervals in the ECG signal for subsequent analysis. The amplitudes and intervals value of $\mathrm{P}-\mathrm{QRS}-\mathrm{T}$ segment determines the functioning of heart of every human. Recently, numerous research and techniques have been developed for analyzing the ECG signal. For subsequent analysis of ECG signals its fundamental features like amplitudes and intervals are required which determine the functioning of heart. The proposed schemes were mostly based on Fuzzy Logic Methods, Artificial Neural Networks (ANN), Genetic Algorithm (GA), Support Vector Machines (SVM), and other Signal Analysis techniques. All these techniques and algorithms have their advantages and limitations. This proposed paper discusses various techniques and transformations proposed earlier in literature for extracting feature from an ECG signal.
\end{abstract}

\section{Keywords}

Artificial Neural Networks (ANN), Cardiac Cycle, ECG signal, Feature Extraction, Fuzzy Logic, Genetic Algorithm

\section{INTRODUCTION}

Electrocardiogram (ECG) is a nearly periodic signal that reflects the activity of the heart. A lot of information on the normal and pathological physiology of heart can be obtained from ECG. However, the ECG signals being nonstationary in nature, it is very difficult to visually analyze them. Thus the need is there for computer based methods for ECG signal Analysis. Clinical observation of ECG can take long hours and can be very tediousECG being a non stationary signal, the irregularities may not be periodic and may show up at different intervals. Moreover, visual analysis cannot be relied upon. This calls for computer based techniques for ECG analysis. At every beat, the heart is depolarized to trigger its contraction. This electrical activity is transmitted throughout the body and can be picked u $\mathrm{p}$ on the skin which is the principle behind the ECG. An ECG machine records this activity via electrodes on the skin and displays it graphically. An ECG involves attaching 10 electrical cables to the body: one to each limb and six across the chest.ECG is a wave that represents an electrical event in the heart such as atria depolarization, ventricular depolarization, atria repolarization, ventricular repolarization. The signal consists of a series of repetitive complex waveforms with a frequency of approximately $1 \mathrm{~Hz}$. One cardiac cycle in an ECG signal consists of the P-QRS-T waves. The majority of the clinically useful information in the ECG is originated in the intervals and amplitudes defined by its features.The improvement of precise and rapid methods for automatic ECG feature extraction is of chief importance, particularly for the examination of long recordings [1].The ECG feature extraction system provides fundamental features (amplitudes and intervals) to be used in subsequent automatic analysis. have been proposed to detect these features [2] [3] [4]. The previously proposed method of ECG signal analysis was based on time domain method. But this is not always adequate to study all the features of ECG signals. Therefore the frequency representation of a signal is required. The deviations in the normal electrical patterns indicate various cardiac disorders.Cardiac cells, in the normal state are electrically polarized [5].previously proposed method of ECG signal analysis was based on time domain method. But this is not always adequate to study all the features of ECG signals. Therefore the frequency representation of a signal is required. The deviations in the normal electrical patterns indicate various cardiac disorders. Cardiac cells, in the normal state are electrically polarized [5].

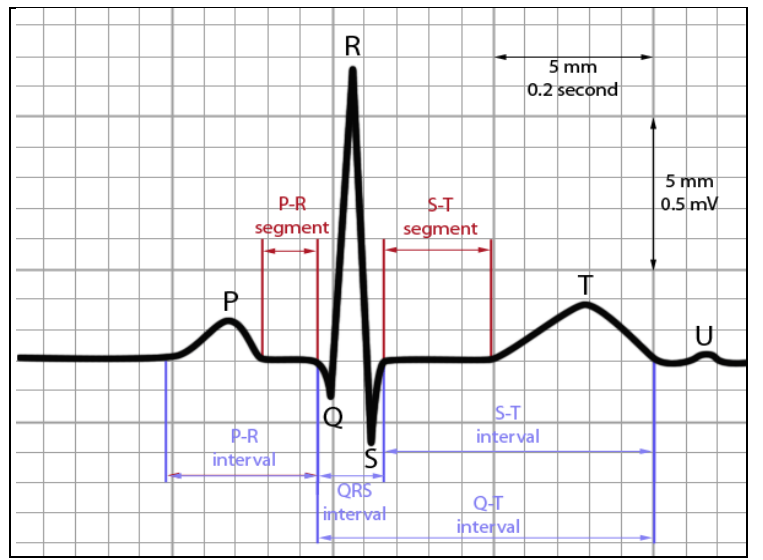

Figure.1 A Sample of ECG Signal showing P-QRS-T Wave

The techniques have been used to address this problem such as the analysis of ECG signals for detection of electrocardiographic changes using the autocorrelation function, frequency domain features, time-frequency analysis, and wavelet transform. Some methods consist of series of band pass filters having frequency range of QRS complexes but these methods have limited accuracy in analyzing ECG features in presence of high frequency noise as well as the ECG signal affected by severe base line drift. In recent years, there has been an increasing interest in applying techniques from the domains of nonlinear analysis and chaos theory in studying the behavior of a dynamical system from an experimental time series such as ECG signal. The purpose of these studies is to determine whether dynamical measures can serve as clinically useful parameters. Various techniques proposed earlier in literature for extracting the features from ECG is analyzed this paper discusses and a review has been made to find out the best 
among them with less computational complexity and more accuracy in prediction and feature extraction.

\section{LITERATURE REVIEW}

ECG feature extraction has been studied from early time and lots of advanced techniques as well as transformations have been proposed for accurate and fast ECG feature extraction. This section of the paper discusses various techniques and transformations proposed earlier in literature for extracting feature from ECG.Zhao et al. [6] proposed a feature extraction method using wavelet transform and support vector machines. The paper presented a new approach to the feature extraction for reliable heart rhythm recognition. The wavelet transform is used to extract the coefficients of the transform as the features of each ECG segment. Concurrently, autoregressive modeling (AR) is also applied to get hold of the temporal structures of ECG waveforms. Then at last the support vector machine (SVM) with Gaussian kernel is used to classify different ECG heart rhythm. The results of computer simulations provided to determine the performance of the proposed approach reached the overall accuracy of $99.68 \%$. Their proposed paper present an algorithm, based on the wavelet transform, for feature extraction from an electrocardiograph (ECG) signal and recognition of abnormal heartbeats. Since wavelet transforms can be localized both in the frequency and time domains. They developed a method for choosing an optimal mother wavelet from a set of orthogonal and bi-orthogonal wavelet filter bank by means of the best correlation with the ECG signal. The foremost step of their approach is to denoise (remove noise) the ECG signal by a soft or hard threshold with limitation of 99.99 reconstructs ability and then each PQRST cycle is decomposed into a coefficients vector by the optimal wavelet function. The coefficients, approximations of the last scale level and the details of the all levels, are used for the ECG analyzed. They divided the coefficients of each cycle into three segments that are related to P-wave, QRS complex, and T-wave. The summation of the values from these segments provided the feature vectors of single cycles. Mahmoodabadi et al. in [1] described an approach for ECG feature extraction which utilizes Daubechies Wavelets transform. They had developed and evaluated an electrocardiogram (ECG) feature extraction system based on the multi-resolution wavelet transform. The ECG signals from Modified Lead II (MLII) were chosen for processing. work is to evaluate the classification performance of an automatic classifier of the electrocardiogram (ECG) for the detection abnormal beats with new concept of feature extraction stage. A classifier was developed with SOM and learning vector quantization (LVQ) algorithms using the data from the records recommended by ANSI/AAMI EC57 standard. In addition their work compares two strategies for classification of annotated QRS complexes: based on original ECG morphology features and proposed new approach - based on preprocessed ECG morphology features. The mathematical morphology filtering is used for the preprocessing of ECG signal.Sufi et al. in [7] formulated a new ECG obfuscation method for feature extraction and corruption detection. They present a new ECG obfuscation method, which uses cross correlation based template matching approach to distinguish all ECG features followed by corruption of those features with added noises. It is extremely difficult to reconstruct the obfuscated features without the knowledge of the templates used for feature matching and the noise. Therefore, they considered three templates and three noises for $\mathrm{P}$ wave, QRS Complex and $\mathrm{T}$ wave comprise the key, which is only $0.4 \%-0.9 \%$ of the original ECG file size. The key distribution among the authorized doctors is efficient and fast because of its small size. To conclude, the experi- ments carried on with imaginably high number of noise combinations the security strength of the presented method was very high. Saxena et al in [9] described an approach for effective feature extraction form ECG signals. Their paper deals with an competent composite method which has been developed for data compression, signal retrieval and feature extraction of ECG signals. After signal retrieval from the compressed data, it has been found that the network not only compresses the data, but also improves the quality of retrieved ECG signal with respect to elimination of high-frequency interference present in the original signal. With the implementation of artificial neural network (ANN) the compression ratio increases as the number of ECG cycle increases. Moreover the features extracted by amplitude, slope and duration criteria from the retrieved signal match with the features of the original signal. Their experimental results at every stage are steady and consistent and prove beyond doubt that the composite method can be used for efficient data management and feature extraction of ECG signals in many real-time applications. A feature extraction method using Discrete Wavelet Transform (DWT) was proposed by Emran et al. in [10]. They used a discrete wavelet transform (DWT) to extract the relevant information from the ECG input data in order to perform the classification task. Their proposed work includes the following modules data acquisition, pre-processing beat detection, feature extraction and classification. In the feature extraction module the Wavelet Transform (DWT) is designed to address the problem of non-stationary ECG signals. It was derived from a single generating function called the mother wavelet by translation and dilation operations. Using DWT infeature extraction may lead to an optimal frequency resolution in all frequency ranges as it has a varying window size, broad at lower frequencies, and narrow at higher frequencies. They DWT characterization will deliver the stable features to the morphology variations of the ECG waveforms. Tayel and Bouridy together in [12] put forth a technique for ECG image classification by extracting their feature using wavelet transformation and neural networks. Features are extracted from wavelet decomposition of the ECG images intensity. The obtained ECG features are then further processed using artificial neural networks. The features are: mean, median, maximum, minimum, range, standard deviation, variance, and mean absolute deviation. The introduced ANN was trained by the main features of the 63 .

ECG images of different diseases. The test results showed that the classification accuracy of the introduced classifier was up to $92 \%$. The extracted features of the ECG signal using wavelet decomposition was effectively utilized by ANN in producing the classification accuracy of $92 \%$.Alan and Nikola in proposed chaos theory that can be successfully applied to ECG feature extraction. They also discussed numerous chaos methods, including phase space and attractors, correlation dimension, spatial filling index, central tendency measure and approximate entropy. They created a new feature extraction environment called ECG chaos extractor to apply the above mentioned chaos methods. A new semi-automatic program for ECG feature extraction has been implemented and is presented in this article[16]. A succession of transformations of the filtered and baseline drift corrected ECG signal is used for mining of a new modified slope-feature. In the presented algorithm, filtering procedure based on moving averages provides smooth spike-free ECG signal, which is appropriate for slope feature extraction. The foremost step is to extort slope feature from the filtered and drift corrected ECG signal, by processing and transforming it, in such a way that the extracted feature signal is significantly enhanced in QRS region and 
suppressed in non-QRS region. The proposed method has detection rate and positive predictivity of $98.56 \%$ and $99.18 \%$ respectively.Xu et al.[16] described an algorithm using Slope Vector Waveform (SVW) for ECG QRS complex detection and RR interval evaluation. In their proposed method variable stage differentiation is used to achieve the desired slope vectors for feature extraction, and the non-linear amplification is used to get better of the signal-to-noise ratio. The method allows for a fast and accurate search of the R location, QRS complex duration, and RR interval and yields excellent ECG feature extraction results. In order to get QRS durations, the feature extraction rules are needed. A method for automatic extraction of both time interval and morphological features, from the Electrocardiogram (ECG) to classify ECGs into normal and arrhythmic was described by Alexakis et al.[12] . The method utilized the combination of artificial neural networks (ANN) and Linear Discriminant Analysis (LDA) techniques for feature extraction. Five ECG features namely RR, RTc, T wave amplitude, T wave skewness, and T wave kurtosis were used in their method. These features are obtained with the assistance of automatic algorithms. The onset and end of the $\mathrm{T}$ wave were detected using the tangent method. The three feature combinations used had very analogous performance when considering the average performance metrics. A modified combined wavelet transforms technique was developed by Saxena et al. The technique has been developed to analyze multi lead electrocardiogram signals for cardiac disease diagnostics. Two wavelets have been used, i.e.a quadratic spline wavelet (QSWT) for QRS detection and the Daubechies six coefficient (DU6) wavelet for P and T detection. A procedure has been evolved using electrocardiogram parameters with a point scoring system for diagnosis of various cardiac diseases. The consistency and reliability of the identified and measured parameters were confirmed when both the diagnostic criteria gave the same results. Table 1 shows the comparison of different ECG signal feature extraction techniques. A robust ECG feature extraction scheme was put forth by Olvera in [12]. The proposed method utilizes a matched filter to detect different signal features on a human heart electrocardiogram signal. The detection of the ST segment, which is a precursor of possible cardiac problems, was more difficult to extract using the matched filter due to noise and amplitude variability. By improving on the methods used; using a different form of the matched filter and better threshold detection, the matched filter ECG feature extraction could be made more successful. The detection of different features in the ECG waveform was much harder than anticipated but it was not due to the implementation of the matched filter. The more complex part was creating the revealing method to remove the feature of interest in each ECG signal. Fatemian et al. [15] proposed an approach for ECG feature extraction.

\section{FUTURE ENHANCEMENT}

The ECG detection which shows the information of the heart and cardiovascular condition is essential to enhance the patient living quality and appropriate treatment. The ECG features can be extracted in time domain[13] or in frequency domain[14]. The extracted feature from the ECG signal plays a vital in diagnosing the cardiac disease. The development of accurate and quick methods for automatic ECG feature extraction is of major importance. Some of the features extraction methods implemented in previous research includes Discrete Wavelet Transform, Karhunen-Loeve Transform, Hermitian Basis and other methods. Every method has its own advantages and limitations. The future work primarily focus on feature extraction from an ECG signal using more statistical data. In addition the future enhancement eye on utilizing dif- ferent transformation technique that provides higher accuracy in feature extraction. The parameters that must be considered while developing an algorithm for feature extraction of an ECG signal are simplicity of the algorithm and the accuracy of the algorithm in providing the best results in feature extraction.

Table I. Comparison of Different Feature Extraction Techniques from an ECG Signal where H, M, L denotes High, Medium and Low respectively

\begin{tabular}{|l|l|l|l|}
\hline Approach & Simplicity & Accuracy & Predictivity \\
\hline Zhao et al & $\mathrm{H}$ & $\mathrm{H}$ & $\mathrm{H}$ \\
\hline $\begin{array}{l}\text { Tadejko } \\
\text { and } \\
\text { Rakowski }\end{array}$ & $\mathrm{L}$ & $\mathrm{M}$ & $\mathrm{M}$ \\
\hline $\begin{array}{l}\text { Alexakis et } \\
\text { al. }\end{array}$ & $\mathrm{H}$ & $\mathrm{M}$ & $\mathrm{M}$ \\
\hline $\begin{array}{l}\text { Xu et al. } \\
\text { Olvera }\end{array}$ & $\mathrm{H}$ & $\mathrm{H}$ & $\mathrm{H}$ \\
\hline Emran et al & $\mathrm{H}$ & $\mathrm{M}$ & $\mathrm{M}$ \\
\hline
\end{tabular}

\section{CONCLUSION}

Various techniques and transformations have been proposed earlier in literature for extracting feature from ECG. This proposed paper provides an over view of various ECG feature extraction techniques and algorithms proposed in literature. The feature extraction technique or algorithm developed for ECG must be highly accurate and should ensure fast extraction of features from the ECG signal. This proposed paper also revealed a comparative table evaluating the performance of different algorithms that were proposed earlier for ECG signal feature extraction. The future work mainly concentrates on developing an algorithm for accurate and fast feature extraction. Therefore our future work also has an eye on improvement in diagnosing the cardiac disease.

\section{REFERENCES}

[1] S. Z. Mahmoodabadi, A. Ahmadian, and M. D. Abolhasani, "ECGFeature Extraction using Daubechies Wavelets," Proceedings of the fifth IASTED International conference on Visualization, Imaging and Image Processing, pp. 343-348, 2005.

[2] Juan Pablo Martínez, Rute Almeida, Salvador Olmos, Ana Paula Rocha,and Pablo Laguna, "A Wavelet-Based ECG Delineator: Evaluation on Standard Databases," IEEE Transactions on Biomedical Engineering Vol. 51, No. 4, pp. 570-581, 2004.

[3] Krishna Prasad and J. S. Sahambi, "Classification of ECG Arrhythmias using Multi-Resolution Analysis and Neural Networks," IEEE Transactions on Biomedical Engineering, vol. 1, pp. 227-231, 2003.

[4] Cuiwei Li, Chongxun Zheng, and Changfeng Tai, "Detection of ECG Characteristic Points using Wavelet Transforms," IEEE Transactions on medical Engineering, Vol. 42, No. 1, pp. 21-28, 1995. 
[5] C. Saritha, V. Sukanya, and Y. Narasimha Murthy, "ECG Signal Analysis Using Wavelet Transforms," Bulgarian Journal of Physics, vol. 35, pp. 68-77, 2008.

[6] Qibin Zhao, and Liqing Zhan, "ECG Feature Extraction and Classification Using Wavelet Transform and Support Vector Machines," International Conference on Neural Networks and Brain, ICNN\&B '05, vol. 2, pp. 10891092, 2005.

[7] B. Castro, D. Kogan, and A. B. Geva, "ECG feature extraction using optimal mother wavelet," The 21st IEEE Convention of the Electrical and Electronic Engineers in Israel, pp. 346-350, 2000.

[8] P. Tadejko, and W. Rakowski, "Mathematical Morphology Based ECG Feature Extraction for the Purpose of Heartbeat Classification," 6thInternational Conference on Computer Information Systems and Industrial Management Applications, CISIM '07, pp. 322-327, 2007.

[9] Felipe E. Olvera, "Electrocardiogram Waveform Feature Extraction Using the Matched Filter," 2006.

[10] Kuo-Kuang Jen, and Yean-Ren Hwang, "ECG Feature Extraction and Classification Using Cepstrum and Neural Networks," Journal of Medical and Biological applications.

[11] S.Pooranchandra, N.Kumaravel, 'A novel method for elimination of power line frequency in ECG signal using hyper shrinkage functions', Digital Signal Processing,Volume18,Issue2, March 2008, pp. 116-126

[12] F. Sufi, S. Mahmoud, I. Khalil, "A new ECG obfuscation method: A joint feature extraction \& corruption approach," International Conference on Information Technology and Applications in Biomedicine, 2008. ITAB 2008, pp. 334-337, May 2008

[13] S. C. Saxena, A. Sharma, and S. C. Chaudhary, "Data compression and feature extraction of ECG signals," International Journal of Systems Science, vol. 28, no. 5, pp. 483-498, 1997.

[14] Emran M. Tamil, Nor Hafeezah Kamarudin, Rosli Salleh, M. Yamani Idna Idris, Noorzaily M.Noor, and Azmi Mohd Tamil, "Heartbeat Electrocardiogram (ECG) Signal Feature Extraction Using Discrete Wavelet Transforms (DWT).'

[15] Mazhar B. Tayel, and Mohamed E. El-Bouridy, "ECG Images Classification Using Feature Extraction Based On Wavelet Transformation And Neural Network," ICGST, International Conference on AIML, June 2006.

[16] Alan Jovic, and Nikola Bogunovic, "Feature Extraction for ECG Time- Series Mining base signal features extraction," 4th National Conference on Telecommunication Technology, 2003. NCTT 2003 Proceedings, pp.232-237, 2003. 